\title{
Relationship between Curriculum-Based Intervention and Fundamental Movement Skills among Preschoolers: A Systematic Literature Review
}

\author{
Zheng Xin, Borhannudin Bin Abdullah*, Nasnoor Juzaily Mohd Nasiruddin, \\ Shamsulariffin Bin Samsudin, Zeinab Zaremohzzabieh
}

Faculty of Educational Studies, Universiti Putra Malaysia, Serdang, 43400, Selangor, Malaysia

Received July 25, 2021; Revised September 18, 2021; Accepted September 27, 2021

\section{Cite This Paper in the following Citation Styles}

(a): [1] Zheng Xin, Borhannudin Bin Abdullah, Nasnoor Juzaily Mohd Nasiruddin, Shamsulariffin Bin Samsudin, Zeinab Zaremohzzabieh, "Relationship between Curriculum-Based Intervention and Fundamental Movement Skills among Preschoolers: A Systematic Literature Review," International Journal of Human Movement and Sports Sciences, Vol. 9, No. 6, pp. 1174 - 1188, 2021. DOI: 10.13189/saj.2021.090612.

(b): Zheng Xin, Borhannudin Bin Abdullah, Nasnoor Juzaily Mohd Nasiruddin, Shamsulariffin Bin Samsudin, Zeinab Zaremohzzabieh (2021). Relationship between Curriculum-Based Intervention and Fundamental Movement Skills among Preschoolers: A Systematic Literature Review. International Journal of Human Movement and Sports Sciences, 9(6), 1174 - 1188. DOI: 10.13189/saj.2021.090612.

Copyright $\odot 2021$ by authors, all rights reserved. Authors agree that this article remains permanently open access under the terms of the Creative Commons Attribution License 4.0 International License

\begin{abstract}
Fundamental movement skills (FMS) are positively correlated with academic achievement, psychology, and participation in physical activities. However, many children's FMS are insufficient, which highlights the need for intervention. The study is aimed at investigating the influence of curriculum foundation intervention on the development of children's FMS and related influencing factors. We systematically reviewed the peer-reviewed articles published across 6 databases (Sport discus, Cochrane Library, PubMed, ScienceDirect, Scopus, Web of Science) on curriculum-based interventions for fundamental movement skills, typically among developing children aged 3-6 years. The risk of bias within the study was assessed using the Quality Assessment and Validity Tool for Correlational Studies (QAVTCS). A total of 14 articles that met the criteria were included, with 1849 participants from across five countries. About $92.9 \%$ of the studies reported a significant improvement in the children's FMS after the intervention. Besides the research report on the instruction strategy, teacher experience, gender, and delivery quality influenced the intervention effect of the children's FMS. In future studies, a more rigorous control design, rich theoretical framework, multi-site study, and different perspectives are needed to identify more influencing factors with regards to intervention and an
\end{abstract}

improvement in the efficacy of the intervention.

Keywords Fundamental Movement Skills, Curriculum, Intervention, Pre-school Children, Pedagogy, Locomotor Skills

\section{Introduction}

Mastering motor competence is a prerequisite for the daily functioning of routines and participation in physical activities, or sports-specific skills later in life [1]. Due to the improvement in people's living standards and the change in their lifestyle, sedentary behavior has increased, and only $26 \%$ of the children's screen times were less than 1 hour per day [2]. In addition, obesity has become a global concern, with 38 million children under the age of 5 recorded as being overweight or obese, in 2021 [3]. Many studies have confirmed that these reasons are closely related to the development of FMS in young children $[4,5]$. There is a lot of evidence that has pointed toward poor FMS proficiencies among young children [68].

Fundamental movement skills (FMS) are associated 
with the basic skills and abilities of a child to perform an organized series of basic movements, which are categorized into three sets: locomotor skills, manipulative skills, and stability skills [1]. A systematic review of the FMS competence by Lubans et al. [9] identified positive correlations with 8 of these variables (self-concept, perceived physical ability, cardio-respiratory fitness CRF, muscle health, weight status, flexibility, physical activity, and reduced sedentary behavior).

Insufficient FMS might set these children up for physical inactivity, and low fitness levels later in their childhood and adulthood [10]. Children who performed poorly on the FMS were more likely to be frustrated, and have trouble acquiring much more complex movement abilities. They may also have low levels of fitness related to health $[11,12]$. They were found to be reluctant to participate in organized sports and competitions [13,14]. Additionally, those who lack FMS had low feelings of self-efficacy and may be less likely to develop an active lifestyle [15].

FMS does not mature with growth or age, and the development of basic motor skills does not occur naturally, but rather, requires the opportunity to practice and carry out appropriate lessons $[16,17]$. The ages of 3-6 have been determined as the critical period for the development of a child's FMS [12,18]. Young children are capable of demonstrating FMS proficiencies by the age of 6 [19]. The capability to learn and master these movements is considered to be key to a child's development, and also their ability to carry out future sports activities. Without early intervention, a child's motor skills development may be delayed, to the extent that it affects them as adults when playing sports. Therefore, to master these skills, specialized and structured exercises should be planned $[18,20]$. To plan successful interventions, the relationship between the intervention and FMA needs to be identified, and it is necessary to detect potential positive factors that affect the acquiring of FMS competence. To help establish a much more informed teaching technique, the FMS intervention strategies need to be understood to achieve a much better outcome.

The Australian government has said that motor skill development and promotion should be an element across all early childhood education programs [21]. China has also issued policies to promote the development of early childhood physical education, and to guide the establishment of early childhood physical education curriculum systems, alongside teacher training systems [22]. Studies over the past 20 years have examined the effectiveness of curriculum-based interventions in improving FMS among preschool children. However, this work has not been consolidated. This study aims to systematically review the research on curricular-based interventions in preschools, to determine the factors which can effectively improve FMS, or affect the intended interventions. It also seeks to understand the effectiveness of these interventions on a child's FMS, in order to improve the ecological validity of these interventions.

\section{Methods}

\subsection{Protocol and Registration}

This review adhered to the Preferred Reporting Items for Systematic Reviews and Meta-analysis (PRISMA) statement guidelines.

\subsection{Eligibility Criteria}

Studies were considered if they fulfilled the following criteria for inclusion: (1) Children ranging in age from 3 to 6 years old, who took part in the study (reflecting preschool); (2) The research was carried out on youngsters who were developing normally, and had no pathological conditions or disabilities; (3) Conducted across a curriculum-based intervention on FMS, and was included in a program plan, or lessons plan; (4) In the presence of assessors or teachers, the FMS was tested using approved techniques; (5) It had been reported that there was a link between FMS and curriculum-based intervention; (6) P-values had been reported; (7) The study included a control group that was comparable in terms of motor competence to that of the intervention group; and (8) The study was not specific to an athletic child, or one with predetermined sporting skills.

\subsection{Search Strategy}

The search was conducted using peer-reviewed literature across six databases (SPORTDiscus, Cochrane Library, PubMed, Science Direct, SCOPUS, and Springer) in December 2020. According to the PICO criteria, the search items were then identified for this review; (the full search strategy reported in Table 1), using the search terms ("fundamental movement skill*" OR "fundamental motor skill*") AND (child* OR infant* OR preschool* OR toddler OR kindergarten) AND (curriculum OR course OR program OR plan OR intervention OR teach* OR method OR model). The reference articles, which met the eligibility criteria, were then added accordingly.

Table 1. Search strategy

\begin{tabular}{|c|c|c|c|}
\hline Population & Intervention & Comparison & Outcome \\
\hline Child* $^{*}$ & Intervention & - & $\begin{array}{c}\text { Fundamental } \\
\text { movement skill* }\end{array}$ \\
\hline Infant* & Curriculum & - & $\begin{array}{c}\text { Fundamental } \\
\text { motor skill* }\end{array}$ \\
\hline Preschool* & Course & - & - \\
\hline Kindergarten & Program & - & - \\
\hline toddler & Plan & - & - \\
\hline- & Teach* & - & - \\
\hline- & Method & - & - \\
\hline- & Model & - & - \\
\hline
\end{tabular}




\subsection{Study Selection}

After deleting duplicates using the Zotero 5.0 software according to the pre-determined eligibility criteria, the articles were initially screened by their titles and abstracts. A total of 34 potentially relevant articles were identified and were then screened using the respective full texts. A total of 23 peer-reviewed studies were attained for quality analysis and data extraction. The above procedure was conducted by two authors (ZX and LXL) independently and cross-checked at each step. This provides the opportunity to present dissenting opinions, which were resolved by the third author. An additional paper was obtained through the screened references, which were included after a discussion between the first two authors.

\subsection{Data Collection}

The first author collected data from the selected studies, and the second author verified the data extraction. The authors extracted the following information from each study; authors, publication year, country, research objectives, study design, participants characteristics, details of the intervention (theory, duration, intensity), comparison, FMS assessments, additional assessments, key findings, and limitations.

\subsection{Quality Assessment}

The risk of bias was assessed using the published Quality Assessment and Validity Tool for Correlational Studies (QAVTCS) [23]. A total of 13-checklist items from the 14 points in each study were used to describe the answer, which helped form a dichotomous variable of "YES" (value 1or 2), and "NO" (values 0). According to the assigned scores, the quality of the papers was classified into three categories: low (0-4), medium (5-9), and high (10-14). The summary results of the quality assessment are reported in Table 2. This flowchart shows how information moves through the various stages of a systematic review.

\section{Results}

\subsection{Study Selection}

The electronic search produced 1818 records for selection, of which 647 duplicate records were deleted, and 1171 remaining titles and abstracts were screened. A total of 1138 irrelevant studies were excluded. The remaining 33 articles were selected by reading the full text. 4 papers were excluded as being non-eligible participants, 2 papers did not measure the FMS, 3 papers did not have an intervention, 8 papers were not curriculum-based, and 2 papers were not peer-reviewed. Quality assessments were completed on the remaining 14 articles, of which 13 studies were rated as high quality, and 1 study was rated as medium quality. No study was assessed as being of low quality. As a result, 14 studies were selected for the systematic review. Table 2 used a multivariate nested cohort design illustration for the flow used in the search process, based on the PRISMA statement (see Figure 1).

\subsection{Study Characteristics}

All studies utilized an experimental research design. A total of 10 studies used a cross-sectional design, and 4 studies used a longitudinal design. The studies were published between 2003 and 2020. The relationship between FMS and the curriculum-based intervention had been studied across 5 countries; the USA ( 8 studies), UK (1 study), Iran (1 study), Australia (3 studies), and Finland (1 study). Most of the studies were done in the United States, and Australia. 
Table 2. Summary of the quality assessment

\begin{tabular}{|c|c|c|c|c|c|c|c|c|c|c|c|c|c|c|c|}
\hline NO. & Criteria & $\begin{array}{c}\text { Palmer } \\
{[34]}\end{array}$ & $\begin{array}{c}\text { Foulkes } \\
{[31]}\end{array}$ & $\begin{array}{c}\text { Mostafavi } \\
{[33]}\end{array}$ & $\begin{array}{c}\text { Jones } \\
{[32]}\end{array}$ & $\begin{array}{c}\text { Goodway } \\
{[37]}\end{array}$ & $\begin{array}{c}\text { Iivonen } \\
\text { [29] }\end{array}$ & $\begin{array}{l}\text { Piek } \\
{[28]}\end{array}$ & $\begin{array}{c}\text { Altunsöz } \\
\& \\
\text { Goodway } \\
{[30]} \\
\end{array}$ & $\begin{array}{c}\text { Nathan } \\
{[24]}\end{array}$ & $\begin{array}{c}\text { Brian \& } \\
\text { Taunton } \\
{[38]}\end{array}$ & $\begin{array}{c}\text { Goodway } \\
\text { \& Branta } \\
{[10]}\end{array}$ & $\begin{array}{c}\text { Robinson } \\
{[40]}\end{array}$ & $\begin{array}{c}\text { Brian } \\
{[36]}\end{array}$ & $\begin{array}{c}\text { Brian } \\
{[35]}\end{array}$ \\
\hline 1 & Future studies & 1 & 1 & 1 & 1 & 1 & 1 & 1 & 1 & 1 & 1 & 1 & 1 & 1 & 1 \\
\hline 2 & Probably sampling & 0 & 1 & 1 & 1 & 1 & 1 & 1 & 1 & 1 & 1 & 1 & 1 & 1 & 1 \\
\hline 3 & $\begin{array}{c}\text { Suitable size of the } \\
\text { sample }\end{array}$ & 1 & 1 & 1 & 1 & 1 & 1 & 1 & 1 & 1 & 1 & 1 & 1 & 1 & 1 \\
\hline 4 & $\begin{array}{l}\text { Sample obtained } \\
\text { from more than } \\
\text { one location }\end{array}$ & 0 & 1 & 1 & 1 & 0 & 1 & 1 & 1 & 1 & 0 & 1 & 0 & 0 & 0 \\
\hline 5 & Privacy preserved & 1 & 1 & 1 & 1 & 1 & 1 & 1 & 1 & 1 & 1 & 1 & 1 & 1 & 1 \\
\hline 6 & $\begin{array}{l}\text { Response } \\
\text { rate }>60 \%\end{array}$ & 1 & 1 & 1 & 1 & 0 & 1 & 1 & 1 & 1 & 1 & 1 & 1 & 1 & 1 \\
\hline 7 & $\begin{array}{c}\text { Reliable } \\
\text { measurement of } \\
\text { result(s) }\end{array}$ & 1 & 1 & 1 & 1 & 1 & 1 & 1 & 1 & 1 & 1 & 1 & 1 & 1 & 1 \\
\hline 8 & $\begin{array}{l}\text { Valid measure of } \\
\text { result(s) }\end{array}$ & 1 & 1 & 1 & 1 & 1 & 1 & 1 & 1 & 1 & 1 & 1 & 1 & 1 & 1 \\
\hline 9 & $\begin{array}{l}\text { Valid measure of } \\
\text { independent } \\
\text { variables }\end{array}$ & 1 & 1 & 1 & 1 & 1 & 0 & 1 & 1 & 1 & 1 & 1 & 1 & 1 & 1 \\
\hline 10 & $\begin{array}{l}\text { Life satisfaction } \\
\text { internal } \\
\text { consistency }\end{array}$ & 2 & 2 & 2 & 0 & 2 & 2 & 2 & 2 & 2 & 2 & 2 & 0 & 2 & 2 \\
\hline 11 & Theory driven & 1 & 1 & 1 & 0 & 1 & 1 & 1 & 1 & 1 & 1 & 1 & 1 & 1 & 1 \\
\hline 12 & $\begin{array}{l}\text { Correlation } \\
\text { analysis for } \\
\text { multiple effects }\end{array}$ & 1 & 0 & 1 & 1 & 0 & 1 & 1 & 1 & 1 & 1 & 1 & 0 & 1 & 1 \\
\hline \multirow[t]{2}{*}{13} & $\begin{array}{c}\text { Outliers } \\
\text { management }\end{array}$ & 1 & 1 & 1 & 1 & 1 & 1 & 0 & 0 & 0 & 0 & 0 & 0 & 1 & 0 \\
\hline & Total & 12 & 13 & 14 & 11 & 12 & 13 & 13 & 13 & 13 & 12 & 13 & 9 & 13 & 12 \\
\hline
\end{tabular}




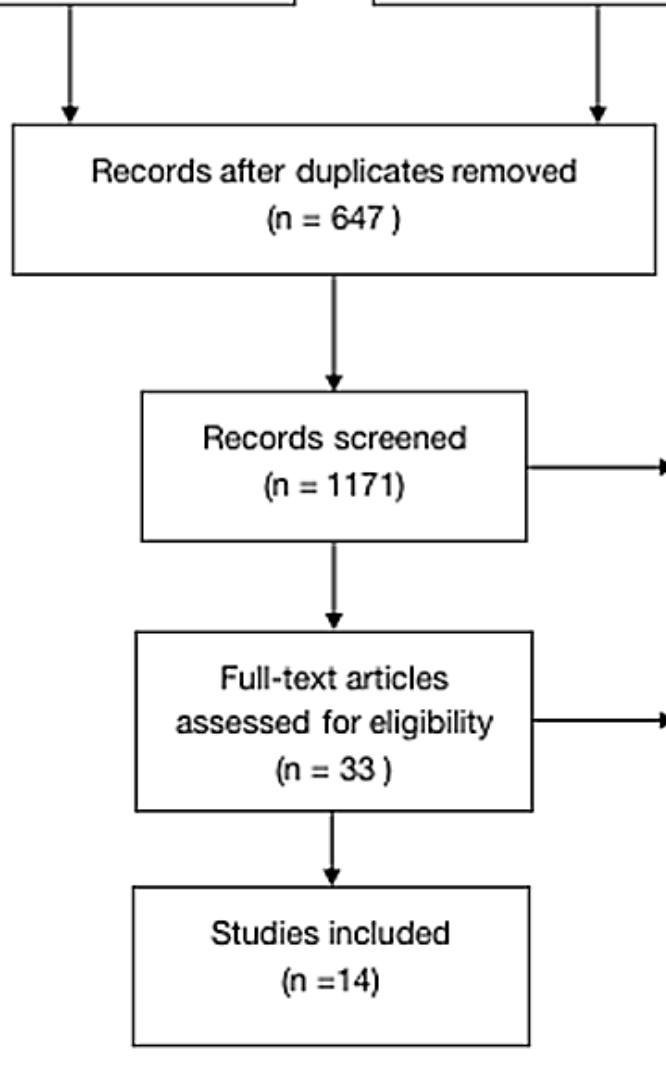

Records excluded

$(n=1138)$

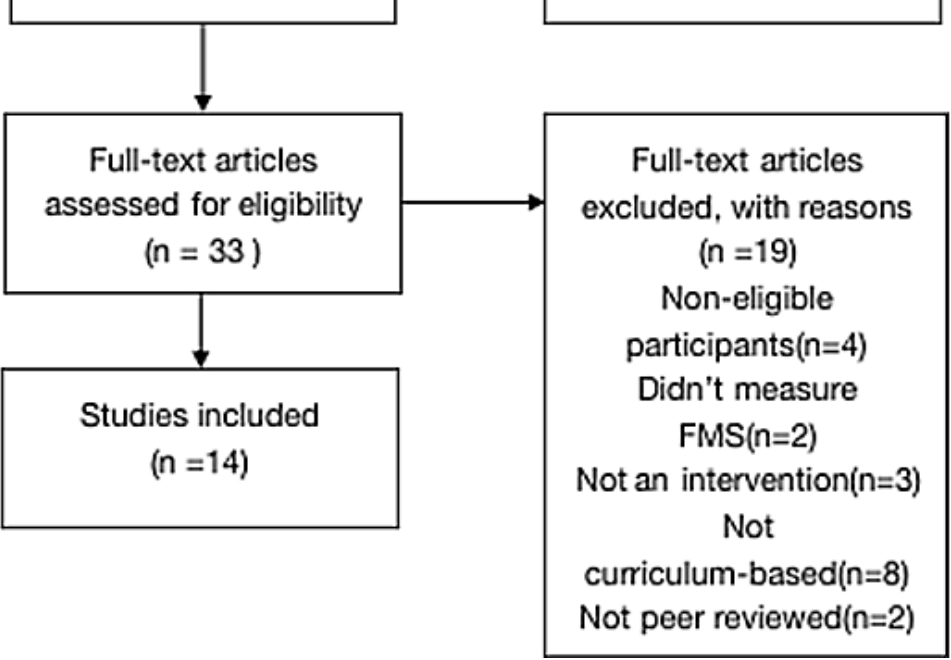

Figure 1. PRISMA flowchart

\section{Participants}

The sample size ranged from 40 to 511, with a total of 1849 participants across all studies. The age of the participants in this study was limited. Each paper reported the age of the participants, and 9 papers reported the mean age. Only one study [24], included kindergarten children as well as children in grades one and two, and the specific number of each grade had not been given. This had an impact on our total statistical number. Excluding that study, the median sample size of the other 13 studies was 90. Overall, in 8 out 14 of the studies, the recruited participants were from low socio-economic areas and reported significantly improved FMS values. The articles reported that the socioeconomic class could affect the physical performance of children, of which, children from low socio-economic backgrounds typically have subordinate levels of FMS development, compared to children from high socioeconomic backgrounds [25-27]. The review focused on developing 3-6-year-old preschoolers, who did not report any specific diseases. A total of 9 out of the 14 studies explicitly reported the sex ratio of the participants and did not have a specify-gender study correlation. Only 3 studies reported the gender differences across FMS. In an article [28], it was claimed that the boys had improved motor skills over time, compared to girls. One article [29], claimed that girls tended to balance their skills developed significantly much more than boys. Altunsöz and Goodway [30] argued that there was a non-significant difference between the genders. Understanding the differences in FMS development between boys and girls could facilitate more targeted interventions in future studies. 
Table 3. An overview of the search protocol based on the PRISMA statemen

\begin{tabular}{|c|c|c|c|c|c|c|c|c|c|c|c|c|c|}
\hline \multirow[t]{2}{*}{ No. } & \multirow[t]{2}{*}{ Author } & \multirow[t]{2}{*}{ Country } & \multirow[t]{2}{*}{ Objectives } & \multirow[t]{2}{*}{ Design } & \multirow[t]{2}{*}{ Participants } & \multicolumn{2}{|c|}{ Intervention } & \multirow[t]{2}{*}{ Comparison } & \multicolumn{2}{|c|}{ FMS Assessment } & \multirow{2}{*}{$\begin{array}{l}\text { Additional } \\
\text { assessment }\end{array}$} & \multirow[t]{2}{*}{ Findings } & \multirow{2}{*}{$\begin{array}{c}\text { Limitations/ } \\
\text { Flaws }\end{array}$} \\
\hline & & & & & & Theory & Dosage & & Time & Type & & & \\
\hline 1 & $\begin{array}{c}\text { Palmer } \\
{[34]}\end{array}$ & USA & $\begin{array}{c}\text { To see how a } \\
\text { 5-week motor } \\
\text { skill intervention } \\
\text { affected } \\
\text { preschooler's } \\
\text { motor skill } \\
\text { competencies and } \\
\text { PA behaviors, } \\
\text { while they were } \\
\text { engaged in the } \\
\text { intervention or } \\
\text { free play. }\end{array}$ & $\begin{array}{l}\text { randomized } \\
\text { controlled } \\
\text { trial }\end{array}$ & $\begin{array}{c}102 \\
\text { preschoolers } \\
61.8 \% \text { boy ; } \\
\text { mean age }= \\
(4.40 \pm 0.43)\end{array}$ & $\begin{array}{c}\text { CHAMP: Based on } \\
\text { the } \\
\text { accomplishment } \\
\text { goal theory, the } \\
\text { high-autonomy } \\
\text { atmosphere } \\
\text { generated by the } 6 \\
\text { TARGET } \\
\text { structures develops } \\
\text { motor skills } \\
\text { through mastery } \\
\text { (Task, Authority, } \\
\text { Recognition, } \\
\text { Grouping, } \\
\text { Evaluation, and } \\
\text { Time) }\end{array}$ & $\begin{array}{c}5 \text { weeks; } 40 \\
\text { min/day; } 3 \\
\text { days/week }\end{array}$ & $\begin{array}{c}\text { outdoor free } \\
\text { play }(\mathrm{n}=38)\end{array}$ & $\begin{array}{l}\text { Pretest- } \\
\text { posttest }\end{array}$ & TGMD-3 & $\begin{array}{c}\text { PA: } \\
\text { Acceleromet } \\
\text { ers }\end{array}$ & $\begin{array}{l}\text { the } \\
\text { intervention } \\
\text { was effective } \\
\text { at improving } \\
\text { preschoolers' } \\
\text { motor skills }\end{array}$ & $\begin{array}{c}\text { the dose ( } 5 \\
\text { weeks, } 600 \text { min) } \\
\text { may be } \\
\text { insufficient } \\
\text { the weather may } \\
\text { have affected } \\
\text { children's PA }\end{array}$ \\
\hline 2 & $\begin{array}{c}\text { Foulkes } \\
{[31]}\end{array}$ & UK & $\begin{array}{c}\text { To see if an active } \\
\text { play intervention } \\
\text { might help } \\
\text { children with } \\
\text { FMS who were } 3 \\
\text { to } 5 \text { years old and } \\
\text { from low-income } \\
\text { families }\end{array}$ & $\begin{array}{l}\text { cluster-rando } \\
\text { mized } \\
\text { controlled } \\
\text { trial design } \\
\text { RCT }\end{array}$ & $\begin{array}{c}162 \text { children } \\
53.1 \% \text { boys } \\
\quad \text { Mean } \\
\text { age }=4.64 \pm \\
0.58 \text { years })\end{array}$ & $\begin{array}{c}\text { Active Play } \\
\text { intended to } \\
\text { enhance young } \\
\text { children's physical } \\
\text { activity, FMS } \\
\text { competence, } \\
\text { self-confidence, } \\
\text { strength, agility, } \\
\text { coordination, and } \\
\text { balance }\end{array}$ & $\begin{array}{c}6 \text { weeks, } 60 \\
\text { minutes/ } \\
\text { week }\end{array}$ & $\begin{array}{l}\text { received a } \\
\text { resource pack } \\
\text { only, no } \\
\text { session } \\
\text { delivery or } \\
\text { support }\end{array}$ & $\begin{array}{l}\text { baseline, } \\
\text { posttest, } \\
\text { 6-month } \\
\text { follow-up }\end{array}$ & TGMD-2 & $\begin{array}{c}\text { BMI: } \\
\text { calibrated } \\
\text { digital scales } \\
\text { and portable } \\
\text { stadiometer }\end{array}$ & $\begin{array}{l}\text { Because there } \\
\text { were no } \\
\text { substantial } \\
\text { variations } \\
\text { between } \\
\text { groups, the } \\
\text { program must } \\
\text { be revised }\end{array}$ & $\begin{array}{l}\text { the response rate } \\
\text { is } 68 \% \text { at } \\
\text { baseline and } \\
\text { decreased at } \\
\text { posttest and } \\
\text { follow-up }\end{array}$ \\
\hline 3 & $\begin{array}{c}\text { Mostafavi } \\
\text { [33] }\end{array}$ & Iran & $\begin{array}{l}\text { To see how the } \\
\text { SPARK Physical } \\
\text { Education } \\
\text { program affected } \\
\text { FMS in 4- } \\
\text { 6-year-old } \\
\text { children. }\end{array}$ & $\begin{array}{l}\text { randomized } \\
\text { controlled } \\
\text { trial design }\end{array}$ & $\begin{array}{l}90 \text { children } \\
\text { aged } 4 \text { to } 6 \\
\text { years }\end{array}$ & $\begin{array}{l}\text { SPARK: based on } \\
\text { physical education } \\
\text { discipline meant to } \\
\text { promote lifelong } \\
\text { well-being without } \\
\text { losing PA pleasure } \\
\text { or academic } \\
\text { performance }\end{array}$ & $\begin{array}{c}8 \text { weeks,3 } \\
\text { sessions } \\
\text { each week }\end{array}$ & $\begin{array}{l}\text { two groups: } \\
\text { 1-Gymnastics; } \\
\text { 2-Routine } \\
\text { activity }\end{array}$ & $\begin{array}{l}\text { pretest- } \\
\text { posttest }\end{array}$ & TGMD-2 & - & $\begin{array}{l}\text { When } \\
\text { compared to } \\
\text { regular } \\
\text { programs or } \\
\text { gymnastics, } \\
\text { the SPARK } \\
\text { program } \\
\text { demonstrated } \\
\text { better } \\
\text { effectiveness } \\
\text { in promoting } \\
\text { the FMS }\end{array}$ & $\begin{array}{l}\text { The economic } \\
\text { aspect of the } \\
\text { SPARK PE } \\
\text { program is not } \\
\text { considered }\end{array}$ \\
\hline
\end{tabular}


Table 3 Continued

\begin{tabular}{|c|c|c|c|c|c|c|c|c|c|c|c|c|c|}
\hline 4 & Jones [32] & Australia & $\begin{array}{l}\text { To evaluate the } \\
\text { feasibility, } \\
\text { acceptability, and } \\
\text { possible efficacy } \\
\text { of a preschool PA } \\
\text { program }\end{array}$ & $\begin{array}{l}\text { 2-arm parallel } \\
\text { cluster } \\
\text { randomized } \\
\text { controlled } \\
\text { pilot trial }\end{array}$ & $\begin{array}{c}\mathrm{N}=97 ; \text { aged } 3 \\
\text { to } 5 \text { years }\end{array}$ & $\begin{array}{c}\text { Jump Start: } \\
\text { Professional } \\
\text { development for } \\
\text { setting personnel, } \\
\text { as well as planned } \\
\text { and unstructured } \\
\text { classes and } \\
\text { activities for } \\
\text { children }\end{array}$ & $\begin{array}{c}20 \text { weeks, } 3 \\
\text { times a week }\end{array}$ & $\begin{array}{l}\text { usual care } \\
\text { activities: } \\
\text { designated } \\
\text { time outside } \\
\text { for free play }\end{array}$ & $\begin{array}{l}\text { pretest- } \\
\text { posttest }\end{array}$ & TGMD-2 & $\begin{array}{l}\text { PA: } \\
\text { Actigraphy } \\
\text { acceleromet } \\
\text { ers; BMI }\end{array}$ & $\begin{array}{l}\text { A staff-led PA } \\
\text { program in a } \\
\text { preschool } \\
\text { context is } \\
\text { possible, } \\
\text { acceptable, and } \\
\text { potentially } \\
\text { effective }\end{array}$ & $\begin{array}{l}\text { The sample size } \\
\text { was insufficient } \\
\text { to report distinct } \\
\text { trends for boys } \\
\text { and girls }\end{array}$ \\
\hline 5 & $\begin{array}{c}\text { Goodway } \\
\text { [37] }\end{array}$ & USA & $\begin{array}{l}\text { The impact of a } \\
\text { 9-week } \\
\text { instructional } \\
\text { program on the } \\
\text { development of } \\
\text { motor and object } \\
\text { control skills in } \\
\text { preschoolers at } \\
\text { risk of } \\
\text { developmental } \\
\text { delay was studied }\end{array}$ & $\begin{array}{l}\text { a pretest- } \\
\text { posttest } \\
\text { quasi- } \\
\text { experimental } \\
\text { design }\end{array}$ & $\begin{array}{l}\text { 63children; } \\
\text { intervention } \\
\text { group mean } \\
\text { age: } 4.9 \\
\text { years; } \\
\text { control group } \\
\text { mean age: } \\
\text { 5.0 years; } \\
\text { from urban } \\
\text { school }\end{array}$ & $\begin{array}{c}\text { SKIP: The } \\
\text { objective was to } \\
\text { provide } \\
\text { developmentally } \\
\text { appropriate } \\
\text { teaching and } \\
\text { practice to enhance } \\
\text { FMAs }\end{array}$ & $\begin{array}{c}9 \text { weeks, } \\
\text { two days per } \\
\text { week,35- } \\
\text { min lessons }\end{array}$ & $\begin{array}{c}\text { regular Pre-K } \\
\text { program: } \\
\text { outdoor free } \\
\text { play }\end{array}$ & $\begin{array}{l}\text { Pretest- } \\
\text { posttest }\end{array}$ & TGMD & - & $\begin{array}{l}\text { Both motor } \\
\text { and object } \\
\text { control } \\
\text { abilities were } \\
\text { considerably } \\
\text { improved in } \\
\text { the } \\
\text { intervention } \\
\text { group than in } \\
\text { the comparison } \\
\text { group }\end{array}$ & $\begin{array}{l}\text { There are no } \\
\text { processes in } \\
\text { place to follow } \\
\text { up on the } \\
\text { situation. Future } \\
\text { study should seek } \\
\text { to monitor } \\
\text { youngsters who } \\
\text { get such teaching } \\
\text { over time }\end{array}$ \\
\hline 6 & $\begin{array}{c}\text { Iivonen } \\
{[29]}\end{array}$ & Finland & $\begin{array}{l}\text { To investigate the } \\
\text { development of } \\
\text { core motor skills } \\
\text { in both linear and } \\
\text { non-linear ways }\end{array}$ & $\begin{array}{l}\text { randomized } \\
\text { controlled } \\
\text { trial }\end{array}$ & $\begin{array}{c}\mathrm{N}=84 ; 54.8 \% \\
\text { boys, aged } \\
\text { four to five } \\
\text { years from } \\
\text { urban } \\
\text { preschools }\end{array}$ & $\begin{array}{l}\text { The Early Steps } \\
\text { initiative (2004- } \\
\text { 07) of the European } \\
\text { Union's Comenius } \\
\text { program included a } \\
\text { physical education } \\
\text { curriculum (PEC) }\end{array}$ & $\begin{array}{l}\text { eight months } \\
\quad(2160 \\
\text { minutes }) \\
\text { two weekly } \\
45 \text {-minute }\end{array}$ & $\begin{array}{c}\text { one } 60 \text {-minute } \\
\text { PE lesson per } \\
\text { week } \\
\text { (altogether } \\
1440 \text { minutes), } \\
\text { no specific } \\
\text { structured } \\
\text { program }\end{array}$ & $\begin{array}{l}\text { 1baseline, } 2 \\
\text { in the middle } \\
\quad \text { of, } 3 \\
\text { immediately } \\
\text { after, } 4 \text { three } \\
\text { months after }\end{array}$ & $\begin{array}{c}\text { APM } \\
\text { Inventory }\end{array}$ & - & $\begin{array}{l}\text { Multiple time } \\
\text { scales are } \\
\text { involved in } \\
\text { motor } \\
\text { development, } \\
\text { and the PEC } \\
\text { encourages } \\
\text { children's FMS }\end{array}$ & $\begin{array}{c}\text { The sample sizes } \\
\text { in this study are a } \\
\text { limitation }\end{array}$ \\
\hline 7 & $\begin{array}{l}\text { Piek et al. } \\
\text { (2013) }\end{array}$ & Australia & $\begin{array}{l}\text { To assess the } \\
\text { efficacy of the } \\
\text { Animal Fun } \\
\text { program }\end{array}$ & $\begin{array}{l}\text { randomized } \\
\text { controlled } \\
\text { trial }\end{array}$ & $\begin{array}{c}511 \text { children } \\
\text { (50.3\%boy) } \\
\text { aged } 4.83 \\
\text { years to } 6.17 \\
\text { years }(\mathrm{M}= \\
5.42 \text { years, } \\
\mathrm{SD}=3.58 \\
\text { months) } \\
\text { from low } \\
\text { socioeconom } \\
\text { ic areas }\end{array}$ & $\begin{array}{l}\text { Animal Fun } \\
\text { program: by } \\
\text { replicating animal } \\
\text { motions in a } \\
\text { pleasant, inclusive } \\
\text { atmosphere, to } \\
\text { improve both } \\
\text { motor coordination } \\
\text { and social abilities } \\
\text { in young children } \\
\text { aged 4-6 years. }\end{array}$ & $\begin{array}{l}6 \text { months, } \\
\text { four days a } \\
\text { week for } 30 \\
\text { min each } \\
\text { day }\end{array}$ & $\begin{array}{l}\text { no information } \\
\text { provided }\end{array}$ & $\begin{array}{l}\text { pre- } \\
\text { intervention, } \\
6 \text { months } \\
\text { after, and } 18 \\
\text { months after }\end{array}$ & $\begin{array}{c}\text { Bruin inks - } \\
\text { Ossietzky } \\
\text { Test of } \\
\text { Motor } \\
\text { Proficiency- } \\
\text { version } 2 \\
\text { Short Form; } \\
\text { Movement } \\
\text { Assessment } \\
\text { Battery for } \\
\text { Children-ve } \\
\text { rsion 2 }\end{array}$ & - & $\begin{array}{l}\text { The Animal } \\
\text { Fun program } \\
\text { dramatically } \\
\text { enhanced } \\
\text { motor } \\
\text { performance in } \\
\text { boys, but not in } \\
\text { girls }\end{array}$ & $\begin{array}{l}\text { in the } \\
\text { intervention } \\
\text { group had poorer } \\
\text { motor skills at } \\
\text { pre-test than the } \\
\text { control group }\end{array}$ \\
\hline
\end{tabular}




\begin{tabular}{|c|c|c|c|c|c|c|c|c|c|c|c|c|c|}
\hline 8 & $\begin{array}{c}\text { Altunsöz } \\
\text { and } \\
\text { Goodway } \\
{[30]}\end{array}$ & USA & $\begin{array}{l}\text { To investigate the } \\
\text { impact of (SKIP } \\
\text { and SKIP-PI - } \\
\text { SKIP-parent } \\
\text { engagement) on } \\
\text { the OC } \\
\text { competence of } \\
\text { impoverished } \\
\text { preschoolers }\end{array}$ & $\begin{array}{l}\text { a pre-post- } \\
\text { retention } \\
\text { experimental } \\
\text { design }\end{array}$ & $\begin{array}{c}72 \text { children } \\
\text { (50\% boys) } \\
\text { from a large } \\
\text { Midwest } \\
\text { urban city }\end{array}$ & $\begin{array}{l}\text { SKIP stands for } \\
\text { effective } \\
\text { kinesthetic } \\
\text { teaching for } \\
\text { preschoolers, as } \\
\text { well as SKIP-PI, } \\
\text { which stands for } \\
\text { SKIP-parent } \\
\text { participation }\end{array}$ & $\begin{array}{c}8 \text { weeks, } \\
\text { two days per } \\
\text { week, } 30 \\
\text { min }\end{array}$ & $\begin{array}{l}\text { regular Head } \\
\text { Start } \\
\text { curriculum }\end{array}$ & $\begin{array}{l}\text { pretest, } \\
\text { posttest, } \\
\text { retention }\end{array}$ & TGMD-2 & - & $\begin{array}{c}\text { When } \\
\text { high-quality } \\
\text { organized } \\
\text { motor skill } \\
\text { intervention in } \\
\text { the form of } \\
\text { SKIP is } \\
\text { offered, kids } \\
\text { can see } \\
\text { considerable } \\
\text { gains in their } \\
\text { OC skill } \\
\text { development }\end{array}$ & $\begin{array}{l}\text { The hardest part } \\
\text { about SKIP-PI is } \\
\text { the inability to } \\
\text { control the home } \\
\text { environment and } \\
\text { thus fidelity is } \\
\text { hard to determine }\end{array}$ \\
\hline 9 & $\begin{array}{l}\text { Nathan } \\
{[24]}\end{array}$ & Australia & $\begin{array}{l}\text { To evaluate the } \\
\text { feasibility and } \\
\text { effectiveness of } \\
\text { the Great Leaders } \\
\text { Active Students } \\
\text { (GLASS) } \\
\text { program, a } \\
\text { school-based } \\
\text { peer-led physical } \\
\text { exercise and } \\
\text { object control } \\
\text { skill intervention }\end{array}$ & $\begin{array}{c}\text { a } \\
\text { quasi-experi } \\
\text { mental design }\end{array}$ & $\begin{array}{c}\mathrm{N}=224 \\
\text { students } \\
\text { from } \\
\text { kindergarten, } \\
\text { Grades 1 and } \\
2\end{array}$ & $\begin{array}{l}\text { GLASS program: } \\
\text { developed utilizing } \\
\text { the concepts of } \\
\text { transformational } \\
\text { leadership theory } \\
\text { and based on the } \\
\text { peer leadership } \\
\text { component of the } \\
\text { Supportive } \\
\text { Children's } \\
\text { Outcome using } \\
\text { Rewards, Exercise, } \\
\text { and Skills } \\
\text { (SCORES) } \\
\text { intervention. }\end{array}$ & $\begin{array}{l}10 \text { weeks, } \\
\text { two } \\
\text { 30-minute } \\
\text { per week }\end{array}$ & usual practice & $\begin{array}{l}\text { baseline, } \\
\text { post-test }\end{array}$ & TGMD-3 & $\begin{array}{c}\text { PA: } \\
\text { pedometer }\end{array}$ & $\begin{array}{c}\text { The } \\
\text { intervention } \\
\text { had a } \\
\text { statistically } \\
\text { significant } \\
\text { effect on } \\
\text { children's total } \\
\text { object } \\
\text { handling } \\
\text { abilities. Peer } \\
\text { leadership as } \\
\text { judged by the } \\
\text { teachers has } \\
\text { considerably } \\
\text { improved }\end{array}$ & $\begin{array}{c}\text { Pedometers were } \\
\text { unable to identify } \\
\text { the intensity of } \\
\text { PA; only } \\
\text { school-time PA } \\
\text { was evaluated; } \\
\text { there was no } \\
\text { information on } \\
\text { the program's } \\
\text { acceptance; The } \\
\text { impact of the } \\
\text { peer-leader } \\
\text { intervention on } \\
\text { PA and OC has } \\
\text { never been } \\
\text { quantified }\end{array}$ \\
\hline 10 & $\begin{array}{c}\text { Brian and } \\
\text { Taunton } \\
{[38]}\end{array}$ & USA & $\begin{array}{l}\text { To investigate the } \\
\text { effects of a motor } \\
\text { skill intervention } \\
\text { provided by } \\
\text { experienced and } \\
\text { novice instructors } \\
\text { using a direct or } \\
\text { indirect } \\
\text { pedagogical } \\
\text { method on young } \\
\text { children's object } \\
\text { control and } \\
\text { locomotor skills }\end{array}$ & $\begin{array}{l}\text { a pretest- } \\
\text { posttest, } \\
\text { control } \\
\text { condition, } \\
\text { experimental } \\
\text { design. }\end{array}$ & $\begin{array}{l}(\mathrm{N}=109) \\
\text { aged } 40-67 \\
\text { months } \\
\text { (Mage }=54 \\
\text { months, } \mathrm{SD}= \\
7 \text { months) }\end{array}$ & $\begin{array}{l}\text { SKIP: Student } \\
\text { learning will be } \\
\text { enhanced by a high } \\
\text { frequency of } \\
\text { successful practice } \\
\text { attempts and } \\
\text { positive congruent } \\
\text { feedback in a } \\
\text { friendly } \\
\text { atmosphere with no } \\
\text { gender, racial, or } \\
\text { disability } \\
\text { differences. }\end{array}$ & $\begin{array}{c}6 \text { weeks } \\
(360 \text { min) } \\
\text { twice } \\
\text { weekly }\end{array}$ & $\begin{array}{l}\text { 'business as } \\
\text { usual' free } \\
\text { play }\end{array}$ & $\begin{array}{l}\text { pretest, } \\
\text { posttest }\end{array}$ & TGMD-2 & - & $\begin{array}{c}\text { The } \\
\text { experimental } \\
\text { children or } \\
\text { teachers } \\
\text { improved their } \\
\text { motor abilities } \\
\text { considerably, } \\
\text { but the control } \\
\text { group did not; } \\
\text { expert-direct } \\
\text { showed higher } \\
\text { improvements } \\
\text { than } \\
\text { novice-indirect }\end{array}$ & $\begin{array}{c}\text { Because this } \\
\text { study is taking } \\
\text { place in a } \\
\text { low-income area, } \\
\text { sample sizes are } \\
\text { small }\end{array}$ \\
\hline
\end{tabular}


Table 3 Continued

\begin{tabular}{|c|c|c|c|c|c|c|c|c|c|c|c|c|c|}
\hline 11 & $\begin{array}{c}\text { Goodway } \\
\text { and } \\
\text { Branta } \\
{[10]}\end{array}$ & USA & $\begin{array}{l}\text { To see how motor } \\
\text { skill intervention } \\
\text { affects the } \\
\text { intervention and } \\
\text { control children's } \\
\text { locomotor and } \\
\text { object control } \\
\text { skills }\end{array}$ & $\begin{array}{c}\text { a } \\
\text { quasi-experi } \\
\text { mental, } \\
\text { pre-posttest-i } \\
\text { ntervention } \\
\quad \text { design }\end{array}$ & $\begin{array}{c}\mathrm{n}=59 \\
\text { 4-year-old } \\
\text { children } \\
\text { from urban } \\
\quad \text { city }\end{array}$ & $\begin{array}{l}\text { MSI: evolved from } \\
\text { a preschool } \\
\text { physical education } \\
\text { curriculum; goals } \\
\text { were created } \\
\text { through official and } \\
\text { informal program } \\
\text { design procedures } \\
\text { including teachers, } \\
\text { paraprofessionals, } \\
\text { and administrators }\end{array}$ & $\begin{array}{l}12 \text { weeks, } \\
24,45 \text {-min } \\
\text { motor } \\
\text { sessions }\end{array}$ & $\begin{array}{l}\text { free play or } \\
\text { recess with } \\
\text { limited } \\
\text { equipment }\end{array}$ & $\begin{array}{l}\text { pretest and } \\
\text { the posttest }\end{array}$ & TGMD & - & $\begin{array}{l}\text { Post-interventi } \\
\text { on object } \\
\text { control scores } \\
\text { in the MSI } \\
\text { group were } \\
\text { substantially } \\
\text { higher than in } \\
\text { the Control } \\
\text { group }\end{array}$ & $\begin{array}{l}\text { There isn't } \\
\text { enough } \\
\text { randomization of } \\
\text { students into } \\
\text { groups }\end{array}$ \\
\hline 12 & $\begin{array}{c}\text { Brian } \\
{[36]}\end{array}$ & USA & $\begin{array}{l}\text { To investigate } \\
\text { how SKIP, a } \\
\text { motor skill } \\
\text { program, } \\
\text { influences pupils' } \\
\text { development of } \\
\text { object handling } \\
\text { skills (OC) }\end{array}$ & $\begin{array}{c}\text { a pretest- } \\
\text { posttest } \\
\text { control group } \\
\text { quasi-experi } \\
\text { mental design }\end{array}$ & $\begin{array}{c}\mathrm{N}=57 ; \text { Boys } \\
=53 \% ; \text { Mage } \\
=4.39, \\
\text { Range } 3-6\end{array}$ & $\begin{array}{l}\text { The SKIP program } \\
\text { accommodates } \\
\text { individual } \\
\text { constraints by } \\
\text { modifying } \\
\text { equipment and } \\
\text { developing } \\
\text { developmentally } \\
\text { appropriate } \\
\text { activities that } \\
\text { match the child's } \\
\text { current level of } \\
\text { motor development }\end{array}$ & $\begin{array}{l}6 \text { weeks } \\
(360 \\
\text { minutes }) \\
\text { two times } \\
\text { per week }\end{array}$ & $\begin{array}{l}\text { 'business as } \\
\text { usual' } \\
\text { curriculum: } \\
\text { free play }\end{array}$ & $\begin{array}{l}\text { pretest and } \\
\text { the posttest }\end{array}$ & TGMD-2 & $\begin{array}{l}\text { Lesson plan } \\
\text { fidelity: } \\
\text { check sheet }\end{array}$ & $\begin{array}{l}\text { When } \\
\text { compared to } \\
\text { control } \\
\text { students, SKIP } \\
\text { children } \\
\text { dramatically } \\
\text { improved their } \\
\text { OC abilities. } \\
\text { Teacher } \\
\text { fidelity had a } \\
\text { beneficial } \\
\text { impact on OC } \\
\text { results }\end{array}$ & $\begin{array}{l}\text { lesser dose of } \\
\text { intervention; }\end{array}$ \\
\hline 13 & $\begin{array}{c}\text { Robinson } \\
\text { [40] }\end{array}$ & USA & $\begin{array}{l}\text { To see how the } \\
\text { mastery climate } \\
\text { motor program } \\
\text { (MMC) affects } \\
\text { object control and } \\
\text { perceived } \\
\text { physical } \\
\text { competence }\end{array}$ & $\begin{array}{c}\text { a } \\
\text { pretest-postte } \\
\text { st, control } \\
\text { condition, } \\
\text { experimental } \\
\text { design. }\end{array}$ & $\begin{array}{l}\mathrm{N}=4060 \% \\
\text { boys ( } \mathrm{M} \text { age } \\
=52.48 \\
\text { months, } \mathrm{SD} \\
=5.12 \text { ) from } \\
\text { urban city }\end{array}$ & $\begin{array}{l}\text { TARGET is a } \\
\text { reference to } \\
\text { MMC's OC skill } \\
\text { intervention, which } \\
\text { is based on } \\
\text { accomplishment } \\
\text { motivation theory. }\end{array}$ & $\begin{array}{c}9 \text { weeks } \\
(432 \\
\text { min),30-min } \\
2 \text { days per } \\
\text { week }\end{array}$ & $\begin{array}{c}\text { free-play } \\
\text { recess }\end{array}$ & $\begin{array}{l}\text { pretest and } \\
\text { the posttest }\end{array}$ & TGMD-2 & $\begin{array}{l}\text { perceived } \\
\text { physical } \\
\text { competence: } \\
\text { a 4-point } \\
\text { scale, }\end{array}$ & $\begin{array}{l}\text { Following the } \\
\text { 9-week } \\
\text { follow-up } \\
\text { session, there } \\
\text { were } \\
\text { substantial } \\
\text { gains, and the } \\
\text { children } \\
\text { reported } \\
\text { greater PPC } \\
\text { over time }\end{array}$ & $\begin{array}{l}\text { There was no } \\
\text { information on } \\
\text { the scale of } \\
\text { perceived } \\
\text { physical } \\
\text { competence } \\
\text { because of the } \\
\text { small sample size }\end{array}$ \\
\hline
\end{tabular}


Table 3 Continued

\begin{tabular}{|c|c|c|c|c|c|c|c|c|c|c|c|c|c|}
\hline 14 & Brian [35] & USA & $\begin{array}{l}\text { To determine the } \\
\text { effectiveness of } \\
\text { the teacher-led } \\
\text { SKIP (T-SKIP) } \\
\text { Program on the } \\
\text { OC skills of } \\
\text { preschool boys } \\
\text { and girls who are } \\
\text { socioeconomicall } \\
\text { y disadvantaged, } \\
\text { and to examine } \\
\text { the fidelity of } \\
\text { Head Start } \\
\text { preschool teachers } \\
\text { implementing the } \\
\text { teacher-led SKIP } \\
\text { (T-SKIP) } \\
\text { Program with } \\
\text { ongoing coaching } \\
\text { and support }\end{array}$ & $\begin{array}{l}\text { a pretest- } \\
\text { posttest, } \\
\text { control } \\
\text { condition, } \\
\text { experimental } \\
\text { design. }\end{array}$ & $\begin{array}{c}\mathrm{N}=122, \\
37 \% \text { boys, } \\
\text { Mage }=4.7 \\
\text { years, } \mathrm{SD}= \\
2.4\end{array}$ & $\begin{array}{l}\text { T-SKIP Program: a } \\
\text { modified version of } \\
\text { SKIP developed for } \\
\text { early childhood } \\
\text { instructors with } \\
\text { little or no prior } \\
\text { experience with } \\
\text { motor development } \\
\text { or physical } \\
\text { education }\end{array}$ & $\begin{array}{c}8 \text { weeks } \\
(450 \text { min), } \\
\text { two sessions } \\
\text { per week }\end{array}$ & $\begin{array}{l}\text { the typical } \\
\text { standard of } \\
\text { practice, or } \\
\text { well-equipped } \\
\text { free play }\end{array}$ & $\begin{array}{l}\text { pretest and } \\
\text { posttest }\end{array}$ & TGMD-2 & $\begin{array}{c}\text { T-SKIP } \\
\text { lesson plan } \\
\text { fidelity: a } \\
\text { check sheet }\end{array}$ & $\begin{array}{l}\text { T-SKIP was } \\
\text { successful with } \\
\text { substantial } \\
\text { variations } \\
\text { across groups; } \\
\text { teacher } \\
\text { faithfulness in } \\
\text { applying } \\
\text { T-SKIP was } \\
\text { lower than the } \\
\text { educational } \\
\text { intervention } \\
\text { literature's } \\
\text { criteria }\end{array}$ & $\begin{array}{l}\text { Due to the severe } \\
\text { winter weather, } \\
\text { schools lost days; } \\
\text { the sample size of } \\
\text { instructors was } \\
\text { tiny }\end{array}$ \\
\hline
\end{tabular}




\subsection{Intervention}

\subsubsection{Intervention period}

Intervention periods ranged from 5 weeks to 8 months across all reviewed literature. A total of 10 out of 14 studies had an intervention period of no more than 10 weeks. Except for Foulkes et al's [31] work which determined that the six-week active play intervention was not significantly different, all other studies reported significant differences. Most of these studies only conducted pre-test and post-test on the dependent variables, across the four articles [28-31]. They also had a follow-up test, which was conducted between the $1^{\text {st }}$ month and the $12^{\text {th }}$ month, after the post-test. One study [29] had a measurement in the middle of the intervention period.

\subsection{Study Design}

The six studies used a randomized controlled experimental design [28,29,31-34], while the others used a quasi-experimental study design.

\subsubsection{Intervention program}

Five of the studies were serviced by the same intervention program-SKIP (Successful Kinesthetic Instruction for Preschoolers) program, and offered different focal points [30,35-38]. 2 of the studies were based on TARGET (Task, Authority, Recognition, Grouping, Evaluation, and Time) [39], structures [34,40]. The remaining 7 intervention programs were Active Play Program (APP) [31], Sports, Play and Active Recreation for Kids (SPARK) [33], Jump Start [41], Physical Education Curriculum (PEC)[29], Animal Fun Program (AFP)[28], Motor Skill Intervention (MSI)[10], and GLASS Program [24].

\subsubsection{Theoretical frameworks}

The author identified 5 kinds of theoretical frameworks for designing intervention programs across the 8 studies $[10,24,28,30,34,38,40]$. These were the Dynamical Systems Theory (DST) [42], the Achievement Goal Theory [39], Newell's Constraints Theory [43], the Transformational Leadership Theory [44], and the Ecological Systems Theory (EST)[45].

\subsubsection{Instruction strategy}

The Instruction Strategies were classified as either direct or indirect instruction. Direct instructions were interpreted as an approach to a highly structured learning environment, which was led by a teacher. Indirect instructions were interpreted as an approach of a less structured learning environment, and a portion of the instructional functions was transferred to the learners $[38,46,47]$. 2 out of 14 studies adopted an indirect instruction strategy.

\subsubsection{Comparison}

9 comparison groups from these studies engaged in free-play activities, and 1 study received the same resource pack as the intervention program without professional guidance and support [31]. The control groups from the 2 studies formed the curriculum, and were part of the Physical Education lesson, with no specific structured program [29] and Gymnastics [33], respectively.

\subsubsection{Outcome Assessment}

\subsubsection{FMS assessment}

6 assessment tools for FMS were identified during the review of the literature, and 2 studies used TGMD [10,37]. 9 studies applied TGMD-2 [30,31,33,35,36,38,40,41]. 2 studies were serviced by TGMD-3 [24,34], 1 study used the Bruininks-Ossietzky Test of Motor Proficiency-version 2 Short Form (BOT-2SF), and the Movement Assessment Battery for Children-version 2 (MABC-2)[28]. 1 study used the APM Inventory [29]. TGMD-2 [48] and TGMD-3 [49] were identified based on the assessment tool- TGMD (Test of Gross Motor Development) [50], with different editions. They had evolved, and improved, considerably. In another systematic review [51], it was indicated that all the 5 measurement tools, except for the APM Inventory, had good reports, and the TGMD series assessment tools had the greatest evidence for validity and reliability. The author searched relevant articles in the APM Inventory and found that there were also good test-retest reliability, and internal consistencies [29,52,53].

\subsubsection{Additional assessment}

In addition to measuring FMS, assessments were also carried out across the 7 articles. 2 articles measured BMI [31,32], 3 articles measured PA using accelerometers [24,32,34], 2 articles measured lesson plan fidelity [35,36], and 1 article measured the perceived physical competence using a four-point scale.

\subsection{Relationship between Curriculum-Based Intervention and FMS}

13 out of the 14 studies reported curriculum-based intervention significantly improved the preschooler's fundamental movement skills. Only 1 study [31] observed a trend for beneficial effects on girls' locomotor skills, but the 6-week intervention did not show a significant difference between the groups. The author presupposed that the insufficient dose, staff training or prior experiences, the delivery quality, and the program curriculum itself could be the primary factors.

Each program provided a specific theoretical direction and course implementation plan. The SKIP program focused on highly organized and structured classes to 
cultivate the children's FMS, as well as the teacher's relevant experiences and instruction strategies. This included the fidelity of the implementation of the lesson plan. Animal fun programs emphasized the children's social/emotional development and the development of the children's FMS in a play situation. The SPARK program emphasized the lifelong wellbeing of the individuals, and the fun gained from participating in developing FMS. The CHAMP program and Mastery motivational climate (MMC) program [40] underlined creating a mastery, and high-autonomy climate for the children's FMS learning based on the achievement motivation theory.

\section{Discussion}

This systematic literature review was aimed at examining the relationship between curriculum-based interventions, and fundamental movement skills, in 36-year-old children. The theoretical framework was evident in $57 \%$ of the studies o. The Dynamical Systems Theory (DST) [42], the Achievement Goal Theory [39], and Newell's Constraints Theory [43] are all the most widely-used theoretical frameworks. The DST provided a rationale for the conceptual model of the intervention program. Newell's Constraints Theory [43], expounded the relationship between the variables, and the Achievement Goal Theory described the young children's participation and motivational factors. Each theoretical framework contributed to the formation of a complete rationale for the children's basic motor skills intervention, which can be referred to in future research, according to the research needs. Meanwhile, in addition to the motivation of the children's psychological development characteristics, one should also pay attention to the sequence of physiological movement development, which can make the theoretical system of intervention much more improved, and could also help teachers conceptualize and plan instructions.

$50 \%$ of the studies in this review not only analyzed the relationship between FMS and curriculum-based intervention, but also studied the correlation between gender, PA, perceived physical competence, the teacher's experience, and the teaching instruction strategy, as well as the results. Some researchers tried to give structured instruction to the children's parents, and involved parents in the intervention. The results found that the parent-assisted intervention was enough to provide certain support for the development of the children's FMS [54,55]. One potential strength of a school - based approach is its ability to delineate important activity and participative situations, and thereby ensure that intervention had the best possible chance of improving meaningful outcomes for a child.

Recent research revealed that combining a Healthy Eating program and an organized outdoor Play as a multi-component preventive intervention to improve the children's FMS, demonstrated a favorable effect on obese children [56]. Most of the research in the review of these literature works was on the intervention programs and across free play or routine activities. Many preschools carried out the sport-related classes using the structured curriculum so that researchers could understand the differences between the intervention programs and the strength and weaknesses of these intervention programs. Existing structured physical activity courses were focused on the children's FMS. This was a means of perfecting and improving the development model, and the theoretical system of the structured curriculum.

Most studies of this review used direct instructions in the teaching process. Ruutmann and Kippe [47] claimed that facts, rules, and action sequences should be taught using instructional strategies from direct instructions. Direct instructions are regarded as the greatest pedagogical technique for eliciting maximum student learning in physical education settings [57]. From the research above, it can be seen that direct instructions are more encouraging than indirect instructions in the teaching process associated with children's FMS. In 42.8 percent of the articles reviewed, the instructors were trained before the intervention, which ensured that teachers were not influenced by prior experiences, and had better delivery quality. In $14.2 \%$ of the articles reviewed, the fidelity of the curriculum plan sought to understand if preschool teachers could faithfully teach according to the structured FMS provided. Some scholars also put forward that effective resources and collaborative delivery modes can be provided to much more professional institutions or resources, for such interventions [24]. The author considers that all the above methods can be referred to 'to improve the ecological validity of the intervention'.

\section{Limitations}

To the knowledge of the authors, this is the first study that used a systematic review to summarize the relationship between curriculum interventions, and basic motor skills across children aged 3-6 years, with a focus on the impact of the curriculum intervention on the basic motoring skills in young children, along with its influencing factors. It was strengthened by the use of objective quality assessment measures. One limitation of this study was it did not include doctoral or masters dissertations, and only articles published in English, which may reduce the generality of the outcome. The other limitation is that this study only focused on curriculum-based interventions related to FMS for 3-6-year-old children (reflect kindergarten); some intervention programs, which were conducted in primary schools, and had not been analyzed. If they performed 
well in primary schools, they may also be properly adjusted and applied to kindergarten environments. For example, the GLASS program [24] evolved from the program based on the SCORES program (supporting children's outcomes using rewards, exercise, and skills) [9], which means that a much broader scope should be investigated in future studies.

\section{Recommendations}

Several recommendations for future studies in this area emerged from the synthesis of the findings. Theoretical or conceptual frameworks of the studies provided a rationale for analyzing the relationship between the variables, which should be used continuously. $71.5 \%$ of the studies in the review were cross-sectional in design, and there was a necessity to increase the proportion of the longitudinal studies, which could avoid limiting the generality of the outcomes. All of the studies included in this review were multisite, which should be pursued further since a variety of various settings would increase the validity and generalizability of the study's findings. Only a few papers included in this study had conducted the multi-locus study. The teacher's experiences, delivery approaches, and commitment to the program's implementation can all influence the effectiveness of the intervention. Multi-component curriculum-based interventions will increase the validity and generality of the findings, and enable researchers to gather more about the factors that influence FMS interventions.

\section{Conclusions}

This review acts as a guide or support for teachers or coaches, to improve FMS in young children. Identifying the association between FMS and curriculum factors will help support teachers in understanding how to effectively use the classroom, and to organize the curriculum to improve the FMS in young children. In addition, the findings of this study can be used as a starting point or reference for future studies on the relationship between curricular interventions and FMS in young children. The children's motor development sequences, psychological development characteristics, and the development needs of non-intellectual factors should all be used as the basic framework for the construction of the intervention programs. The curriculum was proven to be an effective carrier of FMS intervention, but the effect of the intervention was also affected by the instructor, teaching strategies, and socio-economic status of the participants.

As such, it is recommended that curricular interventions coupled with continuous support should be made available to support pre-service teachers in integrating teaching strategies that promote FMS. The curricular interventions need to focus on ensuring that all teachers become capable of providing adequate quality physical activity opportunities for young children; age-appropriate knowledge about physical activity concepts and FMS should also be enhanced. Furthermore, it needs to be confirmed if the effects of the course's interventions extend beyond the motor functions to daily activities, participation, and health. Follow-up measurements should be included in future research works, to examine the long-term impact of the curriculum-based treatments.

\section{REFERENCES}

[1] Goodway, J.D.; Ozmun, J.C.; Gallahue, D.L. Understanding Motor Development: Infants, Children, Adolescents, Adults; 8th ed.; Jones \& Bartlett Learning: Burlington, MA, 2019;

[2] Schranz, N.; Olds, T.; Cliff, D.; Davern, M.; Engelen, L.; ., Giles-Corti, B.; Gomersall, S.; Hardy, L.; Hesketh, K.; Hills, A. Results from Australia's 2014 Report Card on Physical Activity for Children and Youth. Journal of Physical Activity and Health 2014, 11, S21-S25.

[3] WHO Obesity and Overweight Available online: https://www.who.int/en/news-room/fact-sheets/detail/obesi ty-and-overweight.

[4] Collins, A.E.; Pakiz, B.; Rock, C.L. Factors Associated with Obesity in Indonesian Adolescents. International Journal of Pediatric Obesity 2008, 3, 58-64.

[5] Lopes, V.P.; Stodden, D.F.; Bianchi, M.M.; Maia, J.A.; Rodrigues, L.P. Correlation between BMI and Motor Coordination in Children. Journal of Science and Medicine in Sport 2012, 15, 38-43.

[6] Hardy, L.; Reinten-Reynolds, T.; Espinel, P.; Zask, A.; Okely, A. Prevalence and Correlates of Low Motor Skill Competency in Australian Children. Journal of Science and Medicine in Sport 2012, 15, S58-S59.

[7] O'Brien, W.; Belton, S.; Issartel, J. Fundamental Movement Skill Proficiency amongst Adolescent Youth. Physical Education and Sport Pedagogy 2016, 21, 557-571.

[8] Spessato, B.C.; Gabbard, C.; Valentini, N.; Rudisill, M. Gender Differences in Brazilian Children's Fundamental Movement Skill Performance. Early Child Development and Care 2013, 183, 916-923.

[9] Lubans, D.R.; Morgan, P.J.; Cliff, D.P.; Barnett, L.M.; Okely, A.D. Fundamental Movement Skills in Children and Adolescents. Sports medicine 2010, 40, 1019-1035.

[10] Goodway, J.D.; Branta, C.F. Influence of a Motor Skill Intervention on Fundamental Motor Skill Development of Disadvantaged Preschool Children. Research quarterly for exercise and sport 2003, 74, 36-46.

[11] Okely, A.D.; Booth, M.L.; Patterson, J.W. Relationship of Cardiorespiratory Endurance to Fundamental Movement Skill Proficiency among Adolescents. Pediatric Exercise Science 2001, 13, 380-391.

[12] Robinson, L.E.; Wadsworth, D.D.; Peoples, C.M. 
Correlates of School-Day Physical Activity in Preschool Students. Research Quarterly for Exercise and Sport 2012, $83,20-26$

[13] Abdullah, B.; Khalid, H.M.; Lokman, N.A.; Hamidi, M.N.H. Differences in Levels of Control Object Skills among Grade 1 Ballet Dancers, Students with Co-Curricular and Students without Co-Curricular. International Journal of Academic Research in Business and Social Sciences 2018, 8, 491-498.

[14] Okely, A.D.; Booth, M.L.; Patterson, J.W. Relationship of Physical Activity to Fundamental Movement Skills among Adolescents. Medicine and Science in Sports and Exercise 2001, 33, 1899-1904.

[15] Cairney, J.; Hay, J.A.; Faught, B.E.; Wade, T.J.; Corna, L.; Flouris, A. Developmental Coordination Disorder, Generalized Self-Efficacy toward Physical Activity, and Participation in Organized and Free Play Activities. The Journal of pediatrics 2005, 147, 515-520.

[16] Barnett, L.M.; Stodden, D.; Cohen, K.E.; Smith, J.J.; Lubans, D.R.; Lenoir, M.; Iivonen, S.; Miller, A.D.; Laukkanen, A.; Dudley, D. Fundamental Movement Skills: An Important Focus. Journal of Teaching in Physical Education 2016, 35, 219-225.

[17] Clark, J.E. From the Beginning: A Developmental Perspective on Movement and Mobility. Quest 2005, 57, $37-45$.

[18] Payne, V.G.; Isaacs, L.D. Human Motor Development: A Lifespan Approach; 9th ed.; Routledge: London \& New York, 2017;

[19] Gallahue, D.L.; Donnelly, F.C. Developmental Physical Education for All Children; 4th ed.; Human Kinetics: Champaign, IL, 2007;

[20] Zask, A.; Barnett, L.M.; Rose, L.; Brooks, L.O. Three-Year Follow-up of an Early Childhood Intervention: Is Movement Skill Sustained? International Journal of Behavioral Nutrition and Physical Activity 2012, 9, 1-9.

[21] The Department of Health Toddlers and Pre-Schoolers: One to Five Years Available online: https://www1.health.gov.au/internet/publications/publishin g.nsf/Content/gug-director-toc $\sim$ gug-physicalactivity gug-p hysicalactivity-toddlers (accessed on 24 June 2021).

[22] Wang, N.; Rahman, M.N.B.A.; Daud, M.A.K.B.M. Diversified Talent Cultivation Mechanism of Early Childhood Physical Education Under the Full-Practice Concept - Oriented by Preschooler Mental Health and Intelligent Teaching. Frontiers in Psychology 2021, 11, 111, doi:10.3389/fpsyg.2020.593063.

[23] Cicolini, G.; Comparcini, D.; Simonetti, V. Workplace Empowerment and Nurses' Job Satisfaction: A Systematic Literature Review. Journal of Nursing Management 2014, $22,855-871$.

[24] Nathan, N.; Sutherland, R.; Beauchamp, M.R.; Cohen, K.; Hulteen, R.M.; Babic, M.; Wolfenden, L.; Lubans, D.R. Feasibility and Efficacy of the Great Leaders Active StudentS (GLASS) Program on Children's Physical Activity and Object Control Skill Competency: A Non-Randomised Trial. Journal of science and medicine in sport 2017, 20, 1081-1086.
[25] Goodway, J.D.; Robinson, L.E.; Crowe, H. Gender Differences in Fundamental Motor Skill Development in Disadvantaged Preschoolers from Two Geographical Regions. Research quarterly for exercise and sport 2010 , $81,17-24$.

[26] Krombholz, H. Physical Performance in Relation to Age, Sex, Social Class and Sports Activities in Kindergarten and Elementary School. Perceptual and motor skills 1997, 84, $1168-1170$

[27] Morley, D.; Till, K.; Ogilvie, P.; Turner, G. Influences of Gender and Socioeconomic Status on the Motor Proficiency of Children in the UK. Human movement science 2015, 44, 150-156.

[28] Piek, J.P.; McLaren, S.; Kane, R.; Jensen, L.; Dender, A.; Roberts, C.; Rooney, R.; Packer, T.; Straker, L. Does the Animal Fun Program Improve Motor Performance in Children Aged 4-6 Years? Human Movement Science 2013, 32, 1086-1096.

[29] Iivonen, S.; Sääkslahti, A.; Nissinen, K. The Development of Fundamental Motor Skills of Four-to Five-Year-Old Preschool Children and the Effects of a Preschool Physical Education Curriculum. Early Child Development and Care 2011, 181, 335-343.

[30] Altunsöz, I.H.; Goodway, J.D. Skiping to Motor Competence: The Influence of Project Successful Kinesthetic Instruction for Preschoolers on Motor Competence of Disadvantaged Preschoolers. Physical Education and Sport Pedagogy 2016, 21, 366-385.

[31] Foulkes, J.D.; Knowles, Z.; Fairclough, S.J.; Stratton, G.; O'Dwyer, M.; Ridgers, N.D.; Foweather, L. Effect of a 6-Week Active Play Intervention on Fundamental Movement Skill Competence of Preschool Children: A Cluster Randomized Controlled Trial. Perceptual and motor skills 2017, 124, 393-412.

[32] Jones, R.A.; Riethmuller, A.; Hesketh, K.; Trezise, J.; Batterham, M.; Okely, A.D. Promoting Fundamental Movement Skill Development and Physical Activity in Early Childhood Settings: A Cluster Randomized Controlled Trial. Pediatric Exercise Science 2011, 23, 600615 .

[33] Mostafavi, R.; Ziaee, V.; Akbari, H.; Haji-Hosseini, S. The Effects of Spark Physical Education Program on Fundamental Motor Skills in 4-6 Year-Old Children. Iranian journal of pediatrics 2013, 23, 216-219.

[34] Palmer, K.K.; Chinn, K.M.; Robinson, L.E. The Effect of the CHAMP Intervention on Fundamental Motor Skills and Outdoor Physical Activity in Preschoolers. Journal of Sport and Health Science 2019, 8, 98-105.

[35] Brian, A.; Goodway, J.D.; Logan, J.A.; Sutherland, S. SKIPing with Head Start Teachers: Influence of T-SKIP on Object-Control Skills. Research Quarterly for Exercise and Sport 2017, 88, 479-491.

[36] Brian, A.; Goodway, J.D.; Logan, J.A.; Sutherland, S. SKIPing with Teachers: An Early Years Motor Skill Intervention. Physical Education and Sport Pedagogy 2017, $22,270-282$.

[37] Goodway, J.D.; Crowe, H.; Ward, P. Effects of Motor Skill Instruction on Fundamental Motor Skill Development. 
Adapted Physical Activity Quarterly 2003, 20, 298-314.

[38] Brian, A.; Taunton, S. Effectiveness of Intervention Varies on Instructor and Pedagogical Strategy. Physical Education and Sport Pedagogy 2018, 23, 222-233.

[39] Ames, C. Classrooms: Goals, Structures, and Student Motivation. Journal of Educational Psychology 1992, 84, 261-271.

[40] Robinson, L.E. Effect of a Mastery Climate Motor Program on Object Control Skills and Perceived Physical Competence in Preschoolers. Research Quarterly for Exercise and Sport 2011, 82, 355-359, doi:10.1080/02701367.2011.10599764.

[41] Jones, N.; White, M.L.; Tofil, N.; Pickens, M.; Youngblood, A.; Zinkan, L.; Baker, M.D. Randomized Trial Comparing Two Mass Casualty Triage Systems (JumpSTART versus SALT) in a Pediatric Simulated Mass Casualty Event. Prehospital Emergency Care 2014, 18, 417-423.

[42] Thelen, E.; Kelso, J.S.; Fogel, A. Self-Organizing Systems and Infant Motor Development. Developmental Review 1987, 7, 39-65.

[43] Newell, K. Constraints on the development of coordination. In Motor development in children: Aspects of coordination and control; Wade, M.G., Whiting, H.T.A., Eds.; Nijhof, 1986.

[44] Beauchamp, M.R.; Morton, K.L. Transformational Teaching and Physical Activity Engagement among Adolescents. Exercise and Sport Sciences Reviews 2011, 39, 133-139.

[45] Bronfenbrenner, U. Making Human Beings Human: Bioecological Perspectives on Human Development; Sage: Thousand Oaks, CA, 2005;

[46] Rink, J. Teaching Physical Education for Learning; McGraw-Hill Higher Education: Boston, MA, 2010;

[47] Rüütmann, T.; Kipper, H. Teaching Strategies for Direct and Indirect Instruction in Teaching Engineering.; IEEE: Piestany, Slovakia, 2011.

[48] Ulrich, D.A. Test of Gross Motor Development 2nd Ed.
Examiner's Manual; Pro-Ed: Austin, TX, 2000;

[49] Ulrich, D.A. The Test of Gross Motor Development-3 (TGMD-3): Administration, Scoring, and International Norms. Spor Bilimleri Dergisi 2013, 24, 27-33.

[50] Ulrich, D.A.; Sanford, C.B. Test of Gross Motor Development; Pro-ed: Austin, TX, 1985;

[51] Eddy, L.H.; Bingham, D.D.; Crossley, K.L.; Shahid, N.F.; Ellingham-Khan, M.; Otteslev, A.; Figueredo, N.S.; Mon-Williams, M.; Hill, L.J. The Validity and Reliability of Observational Assessment Tools Available to Measure Fundamental Movement Skills in School-Age Children: A Systematic Review. PloS one 2020, 15, e0237919.

[52] Laukkanen, A.; Pesola, A.; Havu, M.; Sääkslahti, A.; Finni, T. Relationship between Habitual Physical Activity and Gross Motor Skills Is Multifaceted in 5-to 8-Year-Old Children. Scandinavian journal of medicine \& science in sports 2014, 24, 102-110.

[53] Numminen, P. APM Inventory: Manual and Test Booklet for Assessing Pre-School Children's Perceptual and Basic Motor Skills. Liikunnan ja Kansanterveyden Julkaisuja 1995.

[54] Hamilton, M.; Goodway, J.; Haubenstricker, J. Parent-Assisted Instruction in a Motor Skill Program for at-Risk Preschool Children. Adapted Physical Activity Quarterly 1999, 16, 415-426.

[55] Irmak, H. The Effects of Two Motor Skill Interventions on Preschool Children's Object Control Skills and Their Perceived Motor Competence. PhD Thesis, The Ohio State University: Ohio, OH, 2010.

[56] Ward, D.S.; Welker, E.; Choate, A.; Henderson, K.E.; Lott, M.; Tovar, A.; Wilson, A.; Sallis, J.F. Strength of Obesity Prevention Interventions in Early Care and Education Settings: A Systematic Review. Preventive Medicine 2017, 95, S37-S52.

[57] Miller, J.; Vine, K.; Larkin, D. The Relationship of Process and Product Performance of the Two-Handed Sidearm Strike. Physical Education and Sport Pedagogy 2007, 12, 61-76. 